\title{
A versatile indium trichloride mediated Prins-type reaction to unsaturated heterocycles
}

\author{
Adrian P. Dobbs, ${ }^{\text {a }}$ Sebastien J.J. Guesné, ${ }^{\mathrm{a}}$ Saša Martinovi,${ }^{\mathrm{a}}$ Simon J. Coles ${ }^{\mathrm{b}}$ and \\ Michael B. Hursthouse ${ }^{b}$ \\ ${ }^{a}$ School of Chemistry, University of Exeter, Stocker Road, Exeter EX4 4QD, UK. \\ ${ }^{b}$ EPSRC National Crystallographic Service, Department of Chemistry, University of \\ Southampton, Highfield, Southampton. SO17 1BJ
}

\section{A.Dobbs@exeter.ac.uk}

\section{Supporting Information}

Representative experimental proceedures and spectral data for compounds 2-4 \& 6 and those in Tables 1 and 2 are provided and crystallographic CIF files for compounds $3 \& 6$.

General $\quad$ S2

(土)-Cis-2-Benzhydryl-6-methyl-5,6-dihydro-2H-pyran (2) S2

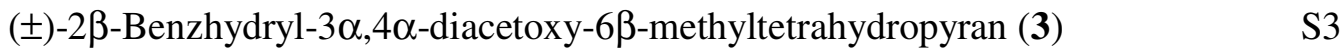

General method for the preparation of thiols (4) from alcohols $\quad$ S4

$\begin{array}{ll}\text { Z-4-Trimethylsilylbut-3-en-1-thiol } & \text { S5 }\end{array}$

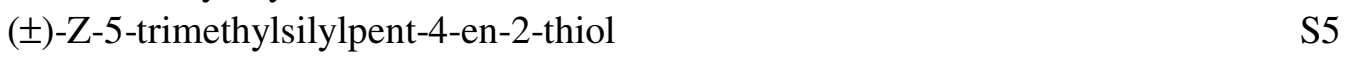

General method for cyclisation reactions of aldehydes with Z-vinylsilyl thiols $\quad$ S6

$\begin{array}{ll}( \pm) \text {-2-Benzyl-5,6-dihydro-2 } H \text {-thiopyran (Table } 1 \text { entry } 1) & \text { S7 }\end{array}$

( \pm )-2-Pentyl-5,6-dihydro-2H-thiopyran (Table 1 entry 2) $\quad$ S7

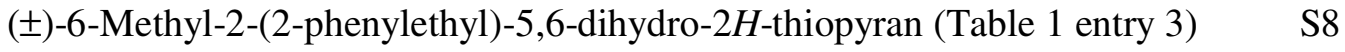

( \pm )-6-Methyl-2-pentyl-5,6-dihydro-2H-thiopyran (Table 1 entry 4) S8

General procedure for the cyclisation of homoallyl amines with aldehydes $\quad$ S9

( \pm )-1,6-Dibenzyl-1,2,3,6-tetrahydropyridine (Table 2 entry 1) S9

( \pm )-6-Pentyl-1-phenyl-1,2,3,6-tetrahydropyridine (Table 2 entry 3) S10

( \pm )-6-Benzyl-1-propyl-1,2,3,6-tetrahydropyridine (Table 2 entry 4) S10

( \pm )-1,6-Dibenzyl-2-methyl-1,2,3,6-tetrahydropyridine (Table 2 entry 5) (6) S11

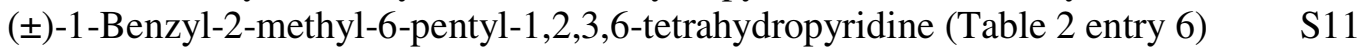

( \pm )-6-Pentyl-2-methyl-1-propyl-1,2,3,6-tetrahydropyridine (Table 2 entry 7) $\quad$ S12

( \pm )-6-Benzyl-2-methyl-1-propyl-1,2,3,6-tetrahydropyridine (Table 2 entry 8) $\quad$ S13

$\begin{array}{lll}{ }^{1} \text { H NMR for ( } \pm \text { )-Cis-2-Benzhydryl-6-methyl-5,6-dihydro-2H-pyran (2) } & \text { S14 }\end{array}$

${ }^{13}$ C NMR for ( \pm )-Cis-2-Benzhydryl-6-methyl-5,6-dihydro-2H-pyran (2) S15

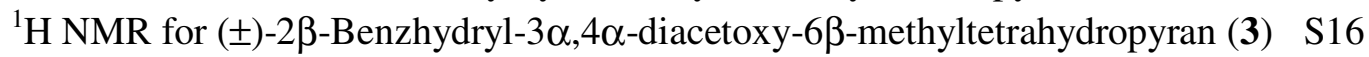

${ }^{13}$ C NMR for $( \pm)-2 \beta$-Benzhydryl-3 $\alpha, 4 \alpha$-diacetoxy-6 $\beta$-methyltetrahydropyran (3) S17

${ }^{1}$ H NMR for ( \pm )-1,6-Dibenzyl-2-methyl-1,2,3,6-tetrahydropyridine (6) $\quad$ S18

${ }^{13} \mathrm{C}$ NMR for $( \pm)$-1,6-Dibenzyl-2-methyl-1,2,3,6-tetrahydropyridine (6) $\quad$ S19 


\section{A. General}

Dichloromethane was distilled over calcium hydride; diethyl ether, THF and toluene were distilled over sodium and benzophenone, which was used as an indicator. All other solvents were obtained anhydrous from Aldrich and used directly into the reaction vessel. All reactions were carried out under an atmosphere of nitrogen unless otherwise stated, using a vacuum/nitrogen manifold. All glassware, syringes and needles were predried in an oven (120-140 $\left.{ }^{\circ} \mathrm{C}\right)$ and cooled in a nitrogen atmosphere prior to use. Stirring was by internal magnetic follower. All chemicals were purified by distillation or recrystallisation where appropriate. Commercially available compounds were generally used without further purification.

All reactions were followed by TLC. Analytical thin layer chromatography was carried out using aluminium backed plates coated with Merck Kieselgel $60 \mathrm{GF}_{254}$. Plates were visualised under UV light (at $254 \mathrm{~nm}$ ) or by staining with acidic ceric ammonium molybdate or acidic potassium permanganate followed by heating. Flash chromatography was carried out using Matrex silica 60, 230-400 mesh; samples were applied as a saturated solution in an appropriate solvent.

Proton $\left({ }^{1} \mathrm{H}\right)$ NMR spectra were recorded at either $300 \mathrm{MHz}$ or $400 \mathrm{MHz}$ and carbon $\left({ }^{13} \mathrm{C}\right)$ NMR spectra at $75 \mathrm{MHz}$ or $100 \mathrm{MHz}$ in deuterated. NMR chemical shifts ( $\delta$ ) are quoted in ppm (parts per million) relative to an internal standard $\left(\mathrm{CDCl}_{3}\right)$. Spectroscopic data is annotated with the following abbreviations: br - broad, $\mathrm{s}$ - singlet, $\mathrm{d}$ - doublet, $\mathrm{t}$ - triplet, and $\mathrm{m}$ - multiplet. Coupling constants are expressed in $\mathrm{Hz} .{ }^{1} \mathrm{H}$ and ${ }^{13} \mathrm{C}$ NMR assignments were made using COSY $\left({ }^{1} \mathrm{H}-{ }^{1} \mathrm{H}\right.$ correlation) and HMQC $\left({ }^{1} \mathrm{H}^{13} \mathrm{C}\right.$ correlation) NMR techniques.

Compounds characterised by high-resolution mass spectrometry were chromatographically homogeneous. Infrared (IR) spectra were recorded in the range $4000-600 \mathrm{~cm}^{-1}$ with internal calibration. Spectra were recorded as $\mathrm{KBr}$ discs or as thin films between $\mathrm{NaCl}$ plates.

\section{( \pm )-Cis-2-Benzhydryl-6-methyl-5,6-dihydro-2H-pyran (2)}

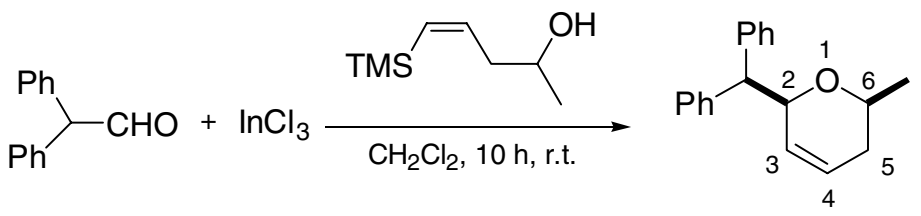


Indium (III) chloride $(0.44 \mathrm{~g}, 2 \mathrm{mmol})$ was added to a $50 \mathrm{ml}$ round bottom flask containing diphenylacetaldehyde (1 eq., $0.39 \mathrm{~g}, 2 \mathrm{mmol})$ dissolved in dry DCM (20 ml) under an atmosphere of nitrogen and the resulting solution was stirred for 1 hour. After this time Z-5-trimethylsilylpent-4-en-2-ol (1 eq., 0.32g, $2 \mathrm{mmol}$ ) was added and the reaction mixture was stirred at room temperature for a further 16 hours. The reaction mixture was then quenched with distilled water $(10 \mathrm{ml})$ and the water layer was extracted with dichloromethane $(40 \mathrm{ml})$. The combined organic extracts were dried with magnesium sulfate. The solvent was removed in vacuo and the reaction mixture purified by flash column chromatography (hexane:diethyl ether 10:1) to give the title compound isolated as a colourless solid ( $0.41 \mathrm{~g}, 78 \%) ; \mathrm{R}_{\mathrm{f}} 0.43$ (petrol:diethyl ether 10:1); Mp 75-77 ${ }^{\circ} \mathrm{C}$ (from petrol); Found $[\mathrm{M}+\mathrm{H}]^{+}$265.1590: $\mathrm{C}_{19} \mathrm{H}_{20} \mathrm{O}+\mathrm{H}$ requires 265.1592; Found: $\mathrm{C}$, 86.3; H, 7.7. Calc. for $\mathrm{C}_{19} \mathrm{H}_{20} \mathrm{O}: \mathrm{C}, 86.3 ; \mathrm{H}, 7.6 \% ; v_{\max } / \mathrm{cm}^{-1}(\mathrm{KBr}) 3027$ [Ar(C-H)], 2970 $(\mathrm{OCH}), 1654(\mathrm{C}=\mathrm{C}), 1598,1495,1449,1388,1183(\mathrm{C}-\mathrm{O}), 1086 ; \delta_{\mathrm{H}}\left(400 \mathrm{MHz} ; \mathrm{CDCl}_{3}\right)$ 7.19-7.39 (10H, m, Ar-H), $5.77(1 \mathrm{H}, \mathrm{m}, \mathrm{C}(4) \mathrm{H}), 5.58(1 \mathrm{H}, \mathrm{dt}, J 10.3,1.9, \mathrm{C}(3) \mathrm{H}), 4.87$ $(1 \mathrm{H}, \mathrm{m}, \mathrm{C}(2) \mathrm{H}), 4.02\left(1 \mathrm{H}, \mathrm{d}, J\right.$ 8.1, $\left.\mathrm{Ph}_{2} \mathrm{C} \underline{\mathrm{H}}\right), 3.75(1 \mathrm{H}, \mathrm{m}, \mathrm{C}(6) \mathrm{H}), 1.95\left(2 \mathrm{H}, \mathrm{m}, \mathrm{C}(5) \mathrm{H}_{2}\right)$, $1.23\left(3 \mathrm{H}, \mathrm{d}, J\right.$ 6.4, $\left.\mathrm{C}(6) \mathrm{CH}_{3}\right) ; \delta_{\mathrm{C}}\left(100 \mathrm{MHz} ; \mathrm{CDCl}_{3}\right) 142.5\left(\mathrm{C}_{i p s o}\right), 142.0\left(\mathrm{C}_{i p s o}\right), 127.9-$ 128.9 [overlapping 8×(C-Ar), C(3)H], $126.3(\mathrm{C}(4) \mathrm{H}), 126.1(\mathrm{C}-\mathrm{Ar}), 125.9(\mathrm{C}-\mathrm{Ar}), 77.3$ $(\mathrm{C}(2) \mathrm{H}), 70.3(\mathrm{C}(6) \mathrm{H}), 56.3\left(\mathrm{Ar}_{2} \underline{\mathrm{C}} \mathrm{H}\right), 32.8\left(\mathrm{C}(5) \mathrm{H}_{2}\right), 21.7\left(\mathrm{C}(6) \underline{\mathrm{C}} \mathrm{H}_{3}\right) ; \mathrm{m} / \mathrm{z}(\mathrm{CI}) 265$ $\left[(\mathrm{MH})^{+}, 90 \%\right], 247\left[(\mathrm{MH})^{+}-\mathrm{H}_{2} \mathrm{O}, 100\right]$.

\section{(士)-2 $\beta$-Benzhydryl-3 $\alpha, 4 \alpha$-diacetoxy-6 $\beta$-methyltetrahydropyran (3)}

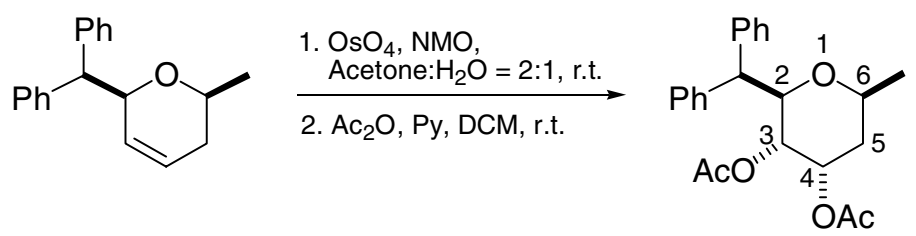

(士)-Cis-2-Benzhydryl-6-methyloxacyclohex-3-ene (0.09 g, $0.3 \mathrm{mmol})$ was placed into a round bottom flask $(25 \mathrm{ml})$ and dissolved in acetone:water 2:1 $(9 \mathrm{ml})$. 4Methylmorpholine $N$-oxide was added $(0.09 \mathrm{~g}, 0.7 \mathrm{mmol}$, 2eq.), followed by two crystals of osmium tetroxide. The flask was sealed with a stopper and the reaction mixture stirred for 48 hours at room temperature. After this time the reaction mixture was cooled to $0{ }^{\circ} \mathrm{C}$, saturated aqueous sodium bisulfite $(6 \mathrm{ml})$ was added and the reaction mixture allowed to 
warm to room temperature. The aqueous layer was extracted with ethyl acetate $(3 \times 20 \mathrm{ml})$. The combined organic layers were washed with brine $(2 \times 10 \mathrm{ml})$, dried over $\mathrm{MgSO}_{4}$,

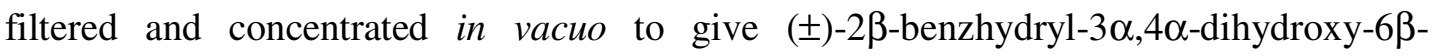
methyloxacyclohexane $(0.06 \mathrm{~g}, 61 \%)$ which was used without further purification. $( \pm)$ $2 \beta$-benzhydryl-3 $\alpha, 4 \alpha$-dihydroxy-6$\beta$-methyloxacyclohexane $(0.06 \mathrm{~g})$ was dissolved in pyridine:DCM 1:1 $(10 \mathrm{ml})$, acetic anhydride was added $(0.61 \mathrm{ml}, 6.4 \mathrm{mmol}, 16$ eq. $)$ and the reaction mixture was stirred overnight (19 hours). After this time the reaction mixture was quenched with saturated aqueous sodium hydrogencarbonate solution $(5 \mathrm{ml})$, the layers were separated and the aqueous layer was extracted with DCM $(3 \times 10 \mathrm{ml})$. The organic layer was washed with $2 \mathrm{M}$ hydrochloric acid $(2 \times 10 \mathrm{ml})$, saturated brine solution $(10 \mathrm{ml})$ and dried over $\mathrm{MgSO}_{4}$. The solvent was removed in vacuo to give $( \pm)-2 \beta$ -

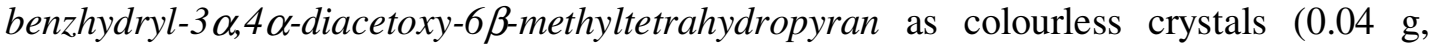
$57 \%$; overall yield 35\%); Mp 144-146 ${ }^{\circ} \mathrm{C}$ (from hexane/diethyl ether); Found $[\mathrm{M}+\mathrm{H}]^{+}$ 383.1851; $\mathrm{C}_{23} \mathrm{H}_{26} \mathrm{O}_{5}+\mathrm{H}$ requires 383.1858; Found: $\mathrm{C}, 72.3$; H, 6.8. Calc. for $\mathrm{C}_{23} \mathrm{H}_{26} \mathrm{O}_{5}$ : C, 72.2; H, 6.8\%; $v_{\max } / \mathrm{cm}^{-1}(\mathrm{KBr}) 3088,3052,3027$ [Ar(C-H)], $2883(\mathrm{OCH}), 1736,(\mathrm{C}=\mathrm{O})$, 1598, 1495, 1449, 1367, 1137 (C-O), 1055; $\delta_{\mathrm{H}}\left(400 \mathrm{MHz} ; \mathrm{CDCl}_{3}\right)$ 7.19-7.44 (10H, m, ArH), $5.40\left(1 \mathrm{H}, \mathrm{m}, \mathrm{Ph}_{2} \mathrm{C} \underline{\mathrm{H}}\right), 4.48(2 \mathrm{H}, \mathrm{m}, \mathrm{C}(2) \mathrm{H}, \mathrm{C}(3) \mathrm{H}), 4.09(1 \mathrm{H}, \mathrm{m}, \mathrm{C}(4) \mathrm{H}), 3.96(1 \mathrm{H}, \mathrm{m}$, $\mathrm{C}(6) \mathrm{H}), 2.11\left(3 \mathrm{H}, \mathrm{s}, \mathrm{OCOCH}_{3}\right), 1.96\left(3 \mathrm{H}, \mathrm{s}, \mathrm{OCOC}_{3}\right), 1.79(1 \mathrm{H}, \mathrm{m}, \mathrm{C}(5) \underline{\mathrm{HH}}), 1.58(1 \mathrm{H}$, m, C(5)H프), $1.20\left(3 \mathrm{H}, \mathrm{d}, J\right.$ 6.2, $\left.\mathrm{C}(6) \mathrm{C}_{3}\right) ; \delta_{\mathrm{C}}\left(100 \mathrm{MHz} ; \mathrm{CDCl}_{3}\right) 170.3\left[\mathrm{C}_{\text {quat }}\left(\mathrm{OCOCH}_{3}\right)\right]$, $169.6\left[\mathrm{C}_{\text {quat }}\left(\mathrm{OCOCH}_{3}\right)\right], 143.1\left(\mathrm{C}_{\text {ipso }}\right), 139.7\left(\mathrm{C}_{i p s o}\right), 130.2$ [overlapping $\left.2 \times \mathrm{C}(\mathrm{Ar})\right], 128.7$ [overlapping $2 \times \mathrm{C}(\mathrm{Ar})$ ], 128.2 [overlapping $2 \times \mathrm{C}(\mathrm{Ar})$ ], 128.0 [overlapping $2 \times \mathrm{C}(\mathrm{Ar})$ ], 126.6 $\mathrm{C}(\mathrm{Ar}), 126.2 \mathrm{C}(\mathrm{Ar}), 74.9$ and $69.8[\mathrm{C}(3$ and 2$) \mathrm{H}], 68.4(\mathrm{C}(6) \mathrm{H}), 67.7\left(\mathrm{Ph}_{2} \mathrm{CH}\right), 51.1$ $(\mathrm{C}(4) \mathrm{H}), 37.4\left(\mathrm{C}(5) \mathrm{H}_{2}\right), 21.1$ [overlapping $\mathrm{C}(3$ and 4$) \mathrm{CHOCOC}_{3}$ ], $20.8\left[\mathrm{C}(6) \underline{\mathrm{CH}}_{3}\right.$ ]; $\mathrm{m} / \mathrm{z}$ (CI) $383\left[(\mathrm{MH})^{+}, 5 \%\right], 323\left[(\mathrm{MH})^{+}-\mathrm{C}_{2} \mathrm{H}_{4} \mathrm{O}_{2}, 10\right], 263\left[(\mathrm{MH})^{+}-2 \times\left(\mathrm{C}_{2} \mathrm{H}_{4} \mathrm{O}_{2}\right), 35\right], 245$ $\left[(\mathrm{MH})^{+}-\mathrm{C}_{4} \mathrm{H}_{10} \mathrm{O}_{5}, 25\right], 215\left[(\mathrm{MH})^{+}-\mathrm{C}_{6} \mathrm{H}_{16} \mathrm{O}_{5}, 25\right], 143\left[(\mathrm{MH})^{+}-\mathrm{C}_{12} \mathrm{H}_{16} \mathrm{O}_{5}, 100\right]$.

\section{General method for the preparation of thiols (4) from alcohols}

Diethyl azodicarboxylate (2 eq., $1.9 \mathrm{ml}, 12 \mathrm{mmol}$ ) was added to a vigorously stirred solution of triphenylphosphine (2 eq., $3.32 \mathrm{~g}, 12 \mathrm{mmol})$ in THF $(100 \mathrm{ml})$ at $0{ }^{\circ} \mathrm{C}$. The mixture was stirred at $0{ }^{\circ} \mathrm{C}$ for 30 minutes. A solution of 4- trimethylsilylbut-3-en-1-ol (1 eq., $6 \mathrm{mmol}$ ) or ( \pm )-Z-5-trimethylsilylpent-4-en-2-ol (1 eq., $6 \mathrm{mmol})$ and thioacetic acid (2 
eq., $0.9 \mathrm{ml}, 12 \mathrm{mmol})$ in THF $(50 \mathrm{ml})$ was added dropwise over 10 minutes and the resulting mixture stirred for 1 hour at $0{ }^{\circ} \mathrm{C}$ and 1 hour at ambient temperature. A clear yellow solution was formed. The solution was concentrated in vacuo and the residue was purified by flash chromatography over silica gel, eluting with petrol:diethyl ether $7: 1$ to give the thioacetate $(0.83 \mathrm{~g}, 61 \%)$. The thioacetate (1 eq., $2 \mathrm{mmol})$ was then dissolved in anhydrous THF (25 ml) and added dropwise to a suspension of lithium aluminium hydride (4 eq., $0.25 \mathrm{~g}, 8 \mathrm{mmol}$ ) in anhydrous ether $(15 \mathrm{ml})$ under a nitrogen atmosphere. The reaction mixture was stirred at ambient temperature for 30 minutes, and the excess lithium aluminium hydride was destroyed by careful addition of $1 \mathrm{M}$ solution of hydrochloric acid $(10 \mathrm{ml})$. The ether layer was separated and dried $\left(\mathrm{MgSO}_{4}\right)$. After evaporation of the solvent, the crude reaction mixture was purified by flash chromatography (petrol:diethyl ether $7: 1$ ) to afford the product.

\section{Z-4-Trimethylsilylbut-3-en-1-thiol}

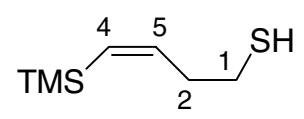

Clear oil

Overall yield for two steps $61 \%$

$\mathrm{R}_{\mathrm{f}} 0.51$ (petrol:ether $7: 1$ )

Found $[\mathrm{M}+\mathrm{H}]^{+} 161.0817 ; \mathrm{C}_{7} \mathrm{H}_{16} \mathrm{SSi}+\mathrm{H}$ requires 161.0821 .

$v_{\max } / \mathrm{cm}^{-1}$ (neat) 2969, $1601(\mathrm{C}=\mathrm{C}), 843(\mathrm{~S}-\mathrm{H}), 687(\mathrm{C}-\mathrm{S})$

$\delta_{\mathrm{H}}\left(300 \mathrm{MHz} ; \mathrm{CDCl}_{3}\right) 6.11(1 \mathrm{H}, \mathrm{dt}, J 14.0,7.2, \mathrm{C}(3) \mathrm{H}), 5.52(1 \mathrm{H}, \mathrm{d}, J$ 14.0, C(4)H), 2.44

$\left(2 \mathrm{H}, \mathrm{m}, \mathrm{C}(1) \mathrm{H}_{2}\right), 2.31\left(2 \mathrm{H}, \mathrm{m}, \mathrm{C}(2) \mathrm{H}_{2}\right), 1.27(1 \mathrm{H}, \mathrm{t}, J$ 7.6, $\mathrm{SH}), 0.06\left(9 \mathrm{H}, \mathrm{s}, 3 \times \mathrm{CH}_{3}\right) ; \delta_{\mathrm{C}}$

$\left(100 \mathrm{MHz} ; \mathrm{CDCl}_{3}\right) 145.3(\mathrm{C}(3) \mathrm{H}), 131.7(\mathrm{C}(4) \mathrm{H}), 37.1\left(\mathrm{C}(1) \mathrm{H}_{2}\right), 24.2\left(\mathrm{C}(2) \mathrm{H}_{2}\right), 0.00$ (TMS).

( \pm )-Z-5-trimethylsilylpent-4-en-2-thiol

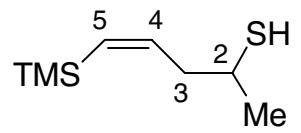

Clear oil 
Overall yield for two steps $56 \%$

$\mathrm{R}_{\mathrm{f}} 0.58$ (petrol:ether $7: 1$ )

Found $[\mathrm{M}+\mathrm{H}]^{+}$175.0961; $\mathrm{C}_{8} \mathrm{H}_{18} \mathrm{SSi}+\mathrm{H}$ requires 175.0977

$\nu_{\max } / \mathrm{cm}^{-1}$ (neat) 2960, $1608(\mathrm{C}=\mathrm{C}), 840(\mathrm{~S}-\mathrm{H}), 686(\mathrm{C}-\mathrm{S})$

$\delta_{\mathrm{H}}\left(300 \mathrm{MHz} ; \mathrm{CDCl}_{3}\right) 6.18(1 \mathrm{H}, \mathrm{ddd}, J 14.1,7.2,6.9, \mathrm{C}(4) \mathrm{H}), 5.51(1 \mathrm{H}, \mathrm{d}, J 14.1, \mathrm{C}(5) \mathrm{H})$, $2.91(1 \mathrm{H}, \mathrm{m}, \mathrm{C}(2) \mathrm{H}), 2.26\left(2 \mathrm{H}, \mathrm{m}, \mathrm{C}(3) \mathrm{H}_{2}\right), 1.47(1 \mathrm{H}, \mathrm{d}, J$ 5.6, SH), $1.20(3 \mathrm{H}, \mathrm{d}, J$ 6.7, $\left.\mathrm{C}(1) \mathrm{H}_{3}\right), 0.02\left(9 \mathrm{H}, \mathrm{s}, 3 \times \mathrm{CH}_{3}\right) ; \delta_{\mathrm{C}}\left(100 \mathrm{MHz} ; \mathrm{CDCl}_{3}\right) 144.9(\mathrm{C}(4) \mathrm{H}), 131.7(\mathrm{C}(5) \mathrm{TMS})$, $43.9\left(\mathrm{C}(3) \mathrm{H}_{2}\right), 35.1(\mathrm{C}(2) \mathrm{H}), 24.6\left(\mathrm{C}(1) \mathrm{H}_{3}\right),-0.21\left(3 \times \mathrm{CH}_{3}\right)$.

$m / z(\mathrm{CI}) 175\left[(\mathrm{MH})^{+}, 70 \%\right], 174\left[(\mathrm{M}-\mathrm{H})^{+}, 100\right], 159\left[(\mathrm{MH})^{+}-\mathrm{CH}_{3}, 55\right], 133\left[(\mathrm{MH})^{+}-\mathrm{C}_{3} \mathrm{H}_{6}\right.$, 75].

\section{General method for cyclisation reactions of aldehydes with $Z$-vinylsilyl thiols}

Indium chloride (1 mmol) was added to a solution of aldehyde (1 mmol) in dry dichloromethane $(20 \mathrm{ml})$, under an atmosphere of nitrogen and the reaction mixture was stirred for 1 hour. After this time, the homoallylic thiol $(1 \mathrm{mmol})$ was added and the reaction mixture stirred at room temperature for a further 5-16 hours. The reaction was monitored by TLC. Upon completion, the reaction mixture was quenched with distilled water $(10 \mathrm{ml})$. The water layer was extracted with dichloromethane $(2 \times 40 \mathrm{ml})$ and the combined organic layer dried with magnesium sulfate. The solvent was removed in vacuo and the reaction mixture purified by flash column chromatography (hexane:diethyl ether 10:1) affording the cyclisation product as an oil. 


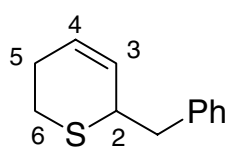

Pale yellow oil.

$51 \%$

$\mathrm{R}_{\mathrm{f}}$ (hexane:diethyl ether 10:1).

Found $[\mathrm{M}+\mathrm{H}]^{+}$191.0888; $\mathrm{C}_{14} \mathrm{H}_{18} \mathrm{~S}+\mathrm{H}$ requires 191.0895 .

$v_{\max } / \mathrm{cm}^{-1}$ (neat) $3015[\mathrm{Ar}(\mathrm{C}-\mathrm{H})], 2834(\mathrm{~S}-\mathrm{CH}), 1651(\mathrm{C}=\mathrm{C}), 1460,741(\mathrm{C}-\mathrm{S}), 696(\mathrm{C}-\mathrm{S})$

$\delta_{\mathrm{H}}\left(400 \mathrm{MHz} ; \mathrm{CDCl}_{3}\right)$ 7.36-7.17 (5H, m, Ar-H), 5.75-5.71 (2H, m, C(3)H \& C(4)H), 3.42

$(1 \mathrm{H}, \mathrm{m}, \mathrm{C}(2) \mathrm{H}), 2.88\left(2 \mathrm{H}, \mathrm{m}, \mathrm{PhCH}_{2}\right), 2.61\left(2 \mathrm{H}, \mathrm{m}, \mathrm{C}(6) \mathrm{H}_{2}\right), 2.23\left(2 \mathrm{H}, \mathrm{m}, \mathrm{C}(5) \mathrm{H}_{2}\right)$.

(士)-2-Pentyl-5,6-dihydro-2H-thiopyran (Table 1 entry 2)

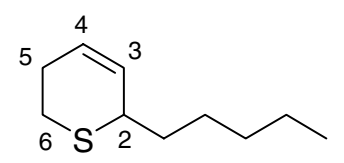

Pale yellow oil

$53 \%$

$\mathrm{R}_{\mathrm{f}} 0.85$ (petrol:diethyl ether 10:1).

Found $[\mathrm{M}+\mathrm{H}]^{+}$171.1199; $\mathrm{C}_{10} \mathrm{H}_{18} \mathrm{~S}+\mathrm{H}$ requires 171.1208 .

$\nu_{\max } / \mathrm{cm}^{-1}$ (neat) 2955, $2858(\mathrm{~S}-\mathrm{CH}), 1649(\mathrm{C}=\mathrm{C}), 700(\mathrm{C}-\mathrm{S})$.

$\delta_{\mathrm{H}}\left(400 \mathrm{MHz} ; \mathrm{CDCl}_{3}\right) 5.71(2 \mathrm{H}, \mathrm{m}, \mathrm{C}(3) \mathrm{H}$ and $\mathrm{C}(4) \mathrm{H}), 3.23(1 \mathrm{H}, \mathrm{m}, \mathrm{C}(2) \mathrm{H}), 2.67(2 \mathrm{H}, \mathrm{m}$, $\left.\mathrm{C}(6) \mathrm{H}_{2}\right), 2.22\left(2 \mathrm{H}, \mathrm{m}, \mathrm{C}(5) \mathrm{H}_{2}\right), 1.51-1.21\left(8 \mathrm{H}, \mathrm{m}\right.$, overlapping $\left.4 \mathrm{xCH}_{2}\right), 0.83(3 \mathrm{H}, \mathrm{t}, J$ 6.8, $\left.\mathrm{CH}_{3}\right)$. 


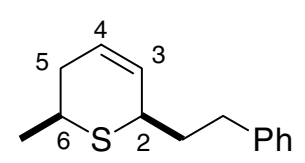

Pale yellow oil.

$68 \%$

$\mathrm{R}_{\mathrm{f}} 0.81$ (hexane:diethyl ether 10:1).

Found $[\mathrm{M}+\mathrm{H}]^{+}$219.1212. $\mathrm{C}_{14} \mathrm{H}_{18} \mathrm{~S}+\mathrm{H}$ requires 219.1207 .

$\nu_{\max } / \mathrm{cm}^{-1}$ (neat) $3021[\operatorname{Ar}(\mathrm{C}-\mathrm{H})], 2824(\mathrm{~S}-\mathrm{CH}), 1644(\mathrm{C}=\mathrm{C}), 1460,1255,737$ (C-S), 697 (C-S).

$\delta_{\mathrm{H}}\left(400 \mathrm{MHz} ; \mathrm{CDCl}_{3}\right)$ 7.19-7.32 (5H, m, Ar-H), $5.82(1 \mathrm{H}, \mathrm{m}, \mathrm{C}(3) \mathrm{H}), 5.72(1 \mathrm{H}, \mathrm{dt}, 12.7$, 1.9, C(4)H), $3.66(1 \mathrm{H}, \mathrm{m}, \mathrm{C}(2) \mathrm{H}), 3.03(1 \mathrm{H}, \mathrm{m}, \mathrm{C}(6) \mathrm{H}), 2.78\left(2 \mathrm{H}, \mathrm{m}, \mathrm{ArC}_{2} \mathrm{CH}_{2}\right), 2.47$ $(1 \mathrm{H}, \mathrm{m}, \mathrm{C}(5) \underline{\mathrm{HH}}), 1.86-2.28\left(3 \mathrm{H}, \mathrm{m}, \mathrm{ArCH}_{2} \underline{\mathrm{CH}}_{2}, \mathrm{C}(5) \mathrm{H} \underline{\mathrm{H}}\right), 1.30\left(3 \mathrm{H}, \mathrm{d}, J 6.8, \mathrm{C}(6) \mathrm{CH}_{3}\right)$; $\delta_{\mathrm{C}}\left(100 \mathrm{MHz} ; \mathrm{CDCl}_{3}\right) 141.7\left(\mathrm{C}_{\text {ipso }}\right), 129.5(\mathrm{C}(4) \mathrm{H}), 128.6$ [overlapping $2 \times \mathrm{C}(\mathrm{Ar})$ ], 127.8 $(\mathrm{C}(3) \mathrm{H}), 127.3(\mathrm{C}(\mathrm{Ar})), 125.9$ [overlapping $2 \times \mathrm{C}(\mathrm{Ar})], 40.1(\mathrm{C}(2) \mathrm{H}), 38.6\left(\mathrm{C}(5) \mathrm{H}_{2}\right), 34.9$ $\left(\mathrm{ArCH}_{2} \mathrm{CH}_{2}\right), 34.5(\mathrm{C}(6) \mathrm{H}), 32.8\left(\mathrm{ArCH}_{2} \underline{\mathrm{CH}}_{2}\right), 21.3\left(\mathrm{C}(6) \underline{\mathrm{CH}}_{3}\right)$.

$\mathrm{m} / \mathrm{z}(\mathrm{CI}) 219\left[(\mathrm{MH})^{+}, 100 \%\right], 127\left[(\mathrm{MH})^{+}-\mathrm{C}_{7} \mathrm{H}_{8}, 10\right]$.

(士)-6-Methyl-2-pentyl-5,6-dihydro-2H-thiopyran (Table 1 entry 4)

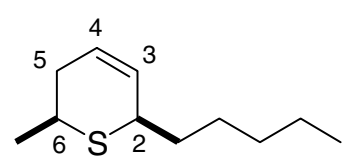

Pale yellow oil

$54 \%$

$\mathrm{R}_{\mathrm{f}} 0.85$ (petrol:diethyl ether 10:1).

Found $[\mathrm{M}+\mathrm{H}]^{+}$185.1365. $\mathrm{C}_{11} \mathrm{H}_{20} \mathrm{~S}+\mathrm{H}$ requires 185.1364 .

$\nu_{\max } / \mathrm{cm}^{-1}$ (neat) 2960, $2852(\mathrm{~S}-\mathrm{CH}), 1654(\mathrm{C}=\mathrm{C}), 702(\mathrm{C}-\mathrm{S})$.

$\delta_{\mathrm{H}}\left(400 \mathrm{MHz} ; \mathrm{CDCl}_{3}\right) 5.75(1 \mathrm{H}, \mathrm{m}, \mathrm{C}(3) \mathrm{H}), 5.67(1 \mathrm{H}, \mathrm{dt}, J$ 12.6, 6.6, C(4)H), $3.61(1 \mathrm{H}, \mathrm{m}$, $\mathrm{C}(2) \mathrm{H}), 3.01(1 \mathrm{H}, \mathrm{m}, \mathrm{C}(6) \mathrm{H}), 2.25(1 \mathrm{H}, \mathrm{m}, \mathrm{C}(5) \mathrm{H}), 1.99(1 \mathrm{H}, \mathrm{m}, \mathrm{C}(5) \mathrm{H}), 1.25-1.62$ 
(overlapping $\left.11 \mathrm{H}, \quad \mathrm{m}, \mathrm{C}(6) \mathrm{CH}_{3}, \mathrm{C}(2) \underline{\mathrm{C}}_{2} \underline{\mathrm{C}}_{2} \underline{\mathrm{C}}_{2} \underline{\mathrm{C}}_{2} \mathrm{CH}_{3}\right), \quad 0.88 \quad(3 \mathrm{H}, \quad \mathrm{t}, \quad J \quad 7.0$, $\left.\mathrm{C}(2) \mathrm{CH}_{2} \mathrm{CH}_{2} \mathrm{CH}_{2} \mathrm{CH}_{2} \mathrm{CH}_{3}\right) ; \delta_{\mathrm{C}}\left(100 \mathrm{MHz} ; \mathrm{CDCl}_{3}\right) 129.9 \mathrm{C}(4) \mathrm{H}, 127.3 \mathrm{C}(3) \mathrm{H}, 40.9 \mathrm{C}(2) \mathrm{H}$, $35.7\left[1 \times \mathrm{CH}_{2}, \mathrm{C}(2) \mathrm{CH}_{2} \mathrm{CH}_{2} \mathrm{CH}_{2} \mathrm{CH}_{2} \mathrm{CH}_{3}\right], 35.0 \mathrm{C}(5) \mathrm{H}_{2}, 34.5 \mathrm{C}(6) \mathrm{H}, 31.7,26.5$ and 22.6 $\left[3 \times \mathrm{CH}_{2}, \mathrm{C}(2) \mathrm{CH}_{2} \mathrm{CH}_{2} \mathrm{CH}_{2} \mathrm{CH}_{2} \mathrm{CH}_{3}\right.$ ], $21.2 \mathrm{C}(6) \underline{\mathrm{CH}}_{3}, 14.1 \mathrm{C}(2) \mathrm{CH}_{2} \mathrm{CH}_{2} \mathrm{CH}_{2} \mathrm{CH}_{2} \mathrm{CH}_{3}$. $m / z(\mathrm{CI}) 185\left[(\mathrm{MH})^{+}, 100 \%\right], 128\left[(\mathrm{MH})^{+}-\mathrm{C}_{4} \mathrm{H}_{9}, 6\right]$.

\section{General procedure for the cyclisation of homoallyl amines with aldehydes}

To a solution of indium trichloride $(221 \mathrm{mg}, 1.0 \mathrm{mmol})$ and aldehyde $(1.0 \mathrm{mmol})$ in anhydrous acetonitrile $(20 \mathrm{~mL})$ at reflux was added dropwise the secondary amine $(1.0$ mmol). Once the reaction was completed (TLC check) the solution was concentrated and the residue obtained partioned between dichloromethane $(20 \mathrm{~mL})$ and $1 \mathrm{M} \mathrm{NaOH}(20$ $\mathrm{mL})$. The aqueous layer was extracted with dichloromethane. The combined organic layers were washed with $1 \mathrm{M} \mathrm{NaOH}$, dried (magnesium sulfate) and concentrated under reduced pressure. The residue was then purified by flash chromatography to give the corresponding tetrahydropyridine.

(士)-1,6-Dibenzyl-1,2,3,6-tetrahydropyridine (Table 2 entry 1)

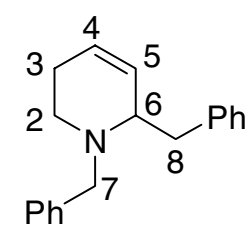

Yellow oil

$95 \%$ yield

$\mathrm{R}_{\mathrm{f}} 0.35$ (hexane:ethyl acetate:triethylamine 94:5:1)

Found: [MH] $]^{+}$264.1752; $\mathrm{C}_{19} \mathrm{H}_{21} \mathrm{~N}+\mathrm{H}$ requires 264.1764 .

$v_{\max } / \mathrm{cm}^{-1}$ (neat) 3084, 3060, 3025, 2921, 2800, 1635, 1602, 1494, 1452, 1363, 728, 698.

$\delta_{\mathrm{H}}\left(400 \mathrm{MHz} ; \mathrm{CDCl}_{3}\right)$ 7.35-7.16 (m, 10H, $\left.\mathrm{H}_{\mathrm{Ph}}\right), 5.76-5.73\left(\mathrm{~m}, 1 \mathrm{H}, \mathrm{H}_{4}\right), 5.53-5.49(\mathrm{~m}, 1 \mathrm{H}$, $\left.\mathrm{H}_{5}\right), 4.00\left(\mathrm{~d}, J 13.2,1 \mathrm{H}, \mathrm{H}_{7}\right), 3.56\left(\mathrm{~d}, J 13.2,1 \mathrm{H}, \mathrm{H}_{7}\right), 3.22-3.18\left(\mathrm{~m}, 1 \mathrm{H}, \mathrm{H}_{6}\right), 3.11(\mathrm{dd}, J$ 5.4 and 13.1, $\left.1 \mathrm{H}, \mathrm{H}_{8}\right), 2.99-2.93\left(\mathrm{~m}, 1 \mathrm{H}, \mathrm{H}_{2}\right), 2.65\left(\mathrm{dd}, J 8.8\right.$ and 13.1, $\left.1 \mathrm{H}, \mathrm{H}_{8}\right), 2.52-2.46$ $\left(\mathrm{m}, 1 \mathrm{H}, \mathrm{H}_{2}\right), 2.12-1.96\left(\mathrm{~m}, 2 \mathrm{H}, \mathrm{H}_{3}\right) ; \delta_{\mathrm{C}}\left(100.6 \mathrm{MHz} ; \mathrm{CDCl}_{3}\right) 139.7\left(\mathrm{C}_{\mathrm{Ph}}\right), 139.5\left(\mathrm{C}_{\mathrm{Ph}}\right)$, 
$129.6\left(\mathrm{C}_{\mathrm{Ph}}\right), 129.1\left(\mathrm{C}_{5}\right), 128.9\left(\mathrm{C}_{\mathrm{Ph}}\right), 128.3\left(\mathrm{C}_{\mathrm{Ph}}\right), 128.2\left(\mathrm{C}_{\mathrm{Ph}}\right), 126.9\left(\mathrm{C}_{\mathrm{Ph}}\right), 126.0\left(\mathrm{C}_{\mathrm{Ph}}\right)$, $125.3\left(\mathrm{C}_{4}\right), 60.5\left(\mathrm{C}_{6}\right), 58.5\left(\mathrm{C}_{7}\right), 45.7\left(\mathrm{C}_{2}\right), 39.7\left(\mathrm{C}_{8}\right), 23.8\left(\mathrm{C}_{3}\right)$.

$\mathrm{m} / \mathrm{z} 264\left(\mathrm{MH}^{+}, 80\right), 172(100), 120(20)$.

(士)-6-Pentyl-1-phenyl-1,2,3,6-tetrahydropyridine (Table 2 entry 3)

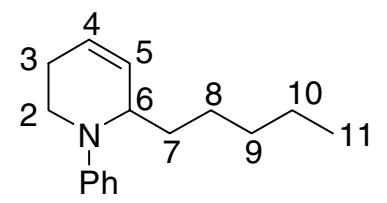

Yellow oil

$95 \%$ yield

$\mathrm{R}_{\mathrm{f}} 0.5$ (hexane)

Found: $[\mathrm{M}+\mathrm{H}]^{+}, 230.1908 . \mathrm{C}_{16} \mathrm{H}_{23} \mathrm{~N}+\mathrm{H}$ requires 230.1908 .

$V_{\max } / \mathrm{cm}^{-1}$ (neat) 3027, 2954, 2927, 1596, 1502, 1457, 1388, 1319, 1247, 746, 692.

$\delta_{\mathrm{H}}\left(400 \mathrm{MHz} ; \mathrm{CDCl}_{3}\right) 7.29-7.34\left(\mathrm{~m}, 2 \mathrm{H}, \mathrm{H}_{\mathrm{Ph}}\right), 6.89\left(\mathrm{~d}, J 7.8,2 \mathrm{H}, \mathrm{H}_{\mathrm{Ph}}\right), 6.78-6.74(\mathrm{~m}, 1 \mathrm{H}$, $\left.\mathrm{H}_{\mathrm{Ph}}\right), 5.91-5.84\left(\mathrm{~m}, 2 \mathrm{H}, \mathrm{H}_{4}\right.$ and $\left.\mathrm{H}_{5}\right), 4.07\left(\mathrm{br} \mathrm{s}, 1 \mathrm{H}, \mathrm{H}_{6}\right), 3.68-3.63\left(\mathrm{~m}, 1 \mathrm{H}, \mathrm{H}_{2}\right), 3.28-3.21$ (m, 1H, $\left.\mathrm{H}_{2}\right), 2.41-2.30\left(\mathrm{~m}, 1 \mathrm{H}, \mathrm{H}_{3}\right), 2.06-1.98\left(\mathrm{~m}, 1 \mathrm{H}, \mathrm{H}_{3}\right), 1.68-1.57\left(\mathrm{~m}, 2 \mathrm{H}, \mathrm{H}_{7}\right), 1.45-$ $1.41\left(\mathrm{~m}, 2 \mathrm{H}, \mathrm{H}_{8}\right), 1.36-1.29\left(\mathrm{~m}, 4 \mathrm{H}, \mathrm{H}_{9}\right.$ and $\left.\mathrm{H}_{10}\right), 0.91\left(\mathrm{t}, J 6.9,3 \mathrm{H}, \mathrm{H}_{11}\right) ; \delta_{\mathrm{C}}(100.6 \mathrm{MHz}$; $\left.\mathrm{CDCl}_{3}\right) 150.3\left(\mathrm{C}_{\mathrm{Ph}}\right), 130.0\left(\mathrm{C}_{5}\right), 129.3\left(\mathrm{C}_{\mathrm{Ph}}\right), 125.5\left(\mathrm{C}_{4}\right), 117.6\left(\mathrm{C}_{\mathrm{Ph}}\right), 115.2\left(\mathrm{C}_{\mathrm{Ph}}\right), 56.3$ $\left(\mathrm{C}_{6}\right), 40.5\left(\mathrm{C}_{2}\right), 32.9\left(\mathrm{C}_{7}\right), 32.2\left(\mathrm{C}_{8}\right), 26.2\left(\mathrm{C}_{9}\right), 24.3\left(\mathrm{C}_{3}\right), 22.8\left(\mathrm{C}_{10}\right), 14.2\left(\mathrm{C}_{11}\right)$ $\mathrm{m} / z 230\left(\mathrm{MH}^{+}, 100\right), 158(31)$

(士)-6-Benzyl-1-propyl-1,2,3,6-tetrahydropyridine (Table 2 entry 4)

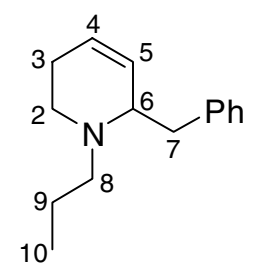

Yellow oil

$55 \%$ yield

$\mathrm{R}_{\mathrm{f}}$ 0.3 (hexane:ethyl acetate:triethylamine 94:5:1)

Found: $[\mathrm{M}+\mathrm{H}]^{+} 216.1752 ; \mathrm{C}_{15} \mathrm{H}_{21} \mathrm{~N}+\mathrm{H}$ requires 216.1770 .

$v_{\max } / \mathrm{cm}^{-1}$ (neat) 3060, 3027, 2958, 2931, 2802, 1604, 1494, 1454, 742, 698. 
$\delta_{\mathrm{H}}\left(400 \mathrm{MHz} ; \mathrm{CDCl}_{3}\right)$ 7.25-7.13 (m, 5H, $\left.\mathrm{H}_{\mathrm{Ph}}\right), 5.70-5.66\left(\mathrm{~m}, 1 \mathrm{H}, \mathrm{H}_{4}\right), 5.43-5.40(\mathrm{~m}, 1 \mathrm{H}$, $\left.\mathrm{H}_{5}\right), 3.12-3.09\left(\mathrm{~m}, 1 \mathrm{H}, \mathrm{H}_{6}\right), 3.02\left(\mathrm{dd}, J 4.6\right.$ and 13.0, 1H, $\left.\mathrm{H}_{7}\right), 2.93(\mathrm{dt}, J 5.8$ and 12.0, 1H, $\left.\mathrm{H}_{2}\right)$, 2.69-2.62 (m, $\left.1 \mathrm{H}, \mathrm{H}_{8}\right), 2.51-2.38\left(\mathrm{~m}, 3 \mathrm{H}, \mathrm{H}_{2} \mathrm{H}_{7}\right.$ and $\left.\mathrm{H}_{8}\right), 2.10-1.98\left(\mathrm{~m}, 2 \mathrm{H}, \mathrm{H}_{3}\right), 1.55-$ $1.44\left(\mathrm{~m}, 2 \mathrm{H}, \mathrm{H}_{9}\right), 0.86\left(\mathrm{t}, J 7.3,3 \mathrm{H}, \mathrm{H}_{10}\right) ; \delta_{\mathrm{C}}\left(100.6 \mathrm{MHz} ; \mathrm{CDCl}_{3}\right) 139.8\left(\mathrm{C}_{\mathrm{Ph}}\right), 129.6\left(\mathrm{C}_{5}\right)$, $129.1\left(\mathrm{C}_{\mathrm{Ph}}\right), 128.3\left(\mathrm{C}_{\mathrm{Ph}}\right), 126.0\left(\mathrm{C}_{4}\right), 125.1\left(\mathrm{C}_{\mathrm{Ph}}\right), 60.7\left(\mathrm{C}_{6}\right), 56.5\left(\mathrm{C}_{8}\right), 46.4\left(\mathrm{C}_{2}\right), 39.4\left(\mathrm{C}_{7}\right)$, $24.5\left(\mathrm{C}_{3}\right), 20.7\left(\mathrm{C}_{9}\right), 12.1\left(\mathrm{C}_{10}\right)$.

$m / z 216\left(\mathrm{MH}^{+}, 55\right), 124(100)$.

(士)-1,6-Dibenzyl-2-methyl-1,2,3,6-tetrahydropyridine (Table 2 entry 5); compound 6

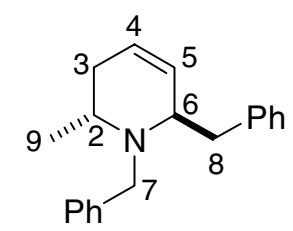

White solid

$68 \%$ yield

$\mathrm{R}_{\mathrm{f}} 0.4$ (hexane:ethyl acetate:triethylamine 94:5:1)

$\mathrm{Mp} 66-67^{\circ} \mathrm{C}$

Found: [M+H[ ${ }^{+} 278.1908 ; \mathrm{C}_{19} \mathrm{H}_{21} \mathrm{~N}+\mathrm{H}$ requires 278.1917 .

$V_{\max } / \mathrm{cm}^{-1}$ (ethyl acetate) 3025, 2962, 2912, 2829, 1739, 1602, 1494, 1452, 1360, 740, 727 , 696.

$\delta_{\mathrm{H}}\left(400 \mathrm{MHz} ; \mathrm{CDCl}_{3}\right)$ 7.26-7.17 (m, 8H, $\left.\mathrm{H}_{\mathrm{Ph}}\right), 7.08-7.06\left(\mathrm{~m}, 2 \mathrm{H}, \mathrm{H}_{\mathrm{Ph}}\right), 5.84-5.81(\mathrm{~m}, 1 \mathrm{H}$, $\left.\mathrm{H}_{4}\right), 5.57-5.53\left(\mathrm{~m}, 1 \mathrm{H}, \mathrm{H}_{5}\right), 3.78\left(\mathrm{~d}, J 14.1,1 \mathrm{H}, \mathrm{H}_{7}\right), 3.55\left(\mathrm{~d}, J 14.1,1 \mathrm{H}, \mathrm{H}_{7}\right), 3.36-3.28$ (m, 1H, $\mathrm{H}_{2}$ ), 3.18 (br s, 1H, $\mathrm{H}_{6}$ ), 2.94 (dd, $J 6.9$ and 13.2, 1H, $\mathrm{H}_{8}$ ), 2.66 (dd, $J 7.7$ and 13.2, $\left.1 \mathrm{H}, \mathrm{H}_{8}\right), 2.06-1.91\left(\mathrm{~m}, 2 \mathrm{H}, \mathrm{H}_{3}\right), 1.18\left(\mathrm{~d}, J\right.$ 6.7, 3H, $\left.\mathrm{H}_{9}\right) ; \delta_{\mathrm{C}}\left(100.6 \mathrm{MHz} ; \mathrm{CDCl}_{3}\right)$ $140.8\left(\mathrm{C}_{\mathrm{Ph}}\right), 140.1\left(\mathrm{C}_{\mathrm{Ph}}\right), 129.7\left(\mathrm{C}_{5}\right), 128.7\left(\mathrm{C}_{\mathrm{Ph}}\right), 128.4\left(\mathrm{C}_{\mathrm{Ph}}\right), 128.1\left(\mathrm{C}_{\mathrm{Ph}}\right), 126.4\left(\mathrm{C}_{\mathrm{Ph}}\right)$, $125.8\left(\mathrm{C}_{\mathrm{Ph}}\right), 125.4\left(\mathrm{C}_{4}\right), 59.3\left(\mathrm{C}_{6}\right), 50.7\left(\mathrm{C}_{7}\right), 47.0\left(\mathrm{C}_{2}\right), 40.7\left(\mathrm{C}_{8}\right), 29.3\left(\mathrm{C}_{3}\right), 17.5\left(\mathrm{C}_{9}\right)$. $m / z 278\left(\mathrm{MH}^{+}, 78\right), 186(100)$.

(士)-1-Benzyl-2-methyl-6-pentyl-1,2,3,6-tetrahydropyridine (Table 2 entry 6)

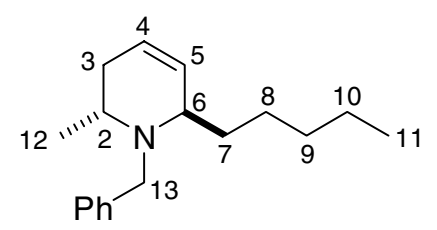

Yellow oil 
$70 \%$ yield

$\mathrm{R}_{\mathrm{f}} 0.3$ (hexane:ethyl acetate:triethylamine 94:5:1)

Found: $[\mathrm{M}+\mathrm{H}]^{+} 258.2222 ; \mathrm{C}_{17} \mathrm{H}_{25} \mathrm{~N}+\mathrm{H}$ requires 258.2229 .

$v_{\text {max }} / \mathrm{cm}^{-1}$ (neat) 3023, 2958, 2927, 2856, 1646, 1456, 1361, 696.

$\delta_{\mathrm{H}}\left(400 \mathrm{MHz} ; \mathrm{CDCl}_{3}\right)$ 7.40-7.38 $\left(\mathrm{m}, 2 \mathrm{H}, \mathrm{H}_{\mathrm{Ph}}\right), 7.32-7.28\left(\mathrm{~m}, 2 \mathrm{H}, \mathrm{H}_{\mathrm{Ph}}\right), 7.26-7.21(\mathrm{~m}, 1 \mathrm{H}$, $\left.\mathrm{H}_{\mathrm{Ph}}\right), 5.80-5.77\left(\mathrm{~m}, 1 \mathrm{H}, \mathrm{H}_{4}\right), 5.63-5.59\left(\mathrm{~m}, 1 \mathrm{H}, \mathrm{H}_{5}\right), 3.71\left(\mathrm{~d}, J 13.8,1 \mathrm{H}, \mathrm{H}_{13}\right), 3.50(\mathrm{~d}, J$ $13.8,1 \mathrm{H}, \mathrm{H}_{13}$ ), 3.18-3.13 (m, 1H, $\mathrm{H}_{2}$ ), 2.93 (br s, 1H, $\mathrm{H}_{6}$ ), 2.06-1.97 (m, 1H, $\mathrm{H}_{3}$ ), 1.92$1.84\left(\mathrm{~m}, 1 \mathrm{H}, \mathrm{H}_{3}\right), 1.54-1.37\left(\mathrm{~m}, 2 \mathrm{H}, \mathrm{H}_{7}\right), 1.36-1.17\left(\mathrm{~m}, 6 \mathrm{H}, \mathrm{H}_{8} \mathrm{H}_{9}\right.$ and $\left.\mathrm{H}_{10}\right), 1.13$ (d, J 6.7, $\left.3 \mathrm{H}, \mathrm{H}_{12}\right), 0.84\left(\mathrm{t}, J\right.$ 7.1, 3H, $\left.\mathrm{H}_{11}\right) ; \delta_{\mathrm{C}}\left(100.6 \mathrm{MHz} ; \mathrm{CDCl}_{3}\right) 141.3\left(\mathrm{C}_{\mathrm{Ph}}\right), 129.8\left(\mathrm{C}_{5}\right), 128.9$ $\left(\mathrm{C}_{\mathrm{Ph}}\right), 128.1\left(\mathrm{C}_{\mathrm{Ph}}\right), 126.5\left(\mathrm{C}_{\mathrm{Ph}}\right), 124.8\left(\mathrm{C}_{4}\right), 56.8\left(\mathrm{C}_{6}\right), 50.8\left(\mathrm{C}_{13}\right), 46.7\left(\mathrm{C}_{2}\right), 33.9\left(\mathrm{C}_{7}\right), 32.0$ $\left(\mathrm{C}_{3}\right), 29.5\left(\mathrm{C}_{8}\right), 25.7\left(\mathrm{C}_{9}\right), 22.8\left(\mathrm{C}_{10}\right), 16.8\left(\mathrm{C}_{12}\right), 14.3\left(\mathrm{C}_{11}\right)$.

$m / z 258\left(\mathrm{MH}^{+}, 98\right), 186(100)$.

(土)-6-Pentyl-2-methyl-1-propyl-1,2,3,6-tetrahydropyridine (Table 2 entry 7)

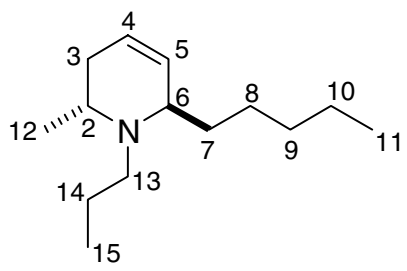

Yellow oil

$73 \%$ yield

$\mathrm{R}_{\mathrm{f}} 0.35$ (hexane:ethyl acetate:triethylamine 94:5:1)

Found: $[\mathrm{M}+\mathrm{H}]^{+} 210.2222 ; \mathrm{C}_{14} \mathrm{H}_{19} \mathrm{~N}+\mathrm{H}$ requires 210.2195 .

$V_{\max } / \mathrm{cm}^{-1}$ (neat) 3020, 2958, 2929, 2871, 1733, 1465, 1375, 1149, 703.

$\delta_{\mathrm{H}}\left(400 \mathrm{MHz} ; \mathrm{CDCl}_{3}\right)$ 5.72-5.69 (m, 1H, $\left.\mathrm{H}_{4}\right), 5.62-5.58\left(\mathrm{~m}, 1 \mathrm{H}, \mathrm{H}_{5}\right), 3.11-3.05(\mathrm{~m}, 1 \mathrm{H}$, $\left.\mathrm{H}_{2}\right), 2.93-2.92\left(\mathrm{~m}, 1 \mathrm{H}, \mathrm{H}_{6}\right), 2.41-2.26\left(\mathrm{~m}, 2 \mathrm{H}, \mathrm{H}_{13}\right), 1.98-1.89\left(\mathrm{~m}, 1 \mathrm{H}, \mathrm{H}_{3}\right), 1.85-1.75(\mathrm{~m}$, $\left.1 \mathrm{H}, \mathrm{H}_{3}\right), 1.44-1.25\left(\mathrm{~m}, 10 \mathrm{H}, \mathrm{H}_{7} \mathrm{H}_{8} \mathrm{H}_{9} \mathrm{H}_{10}\right.$ and $\left.\mathrm{H}_{14}\right), 1.02$ (d, J 6.7, 3H, $\mathrm{H}_{12}$ ), 0.90-0.86 (m, $6 \mathrm{H}, \mathrm{H}_{11}$ and $\left.\mathrm{H}_{15}\right) ; \delta_{\mathrm{C}}\left(100.6 \mathrm{MHz} ; \mathrm{CDCl}_{3}\right) 129.9\left(\mathrm{C}_{5}\right), 124.7\left(\mathrm{C}_{4}\right), 57.7\left(\mathrm{C}_{6}\right), 48.9\left(\mathrm{C}_{13}\right)$, $47.0\left(\mathrm{C}_{2}\right), 33.8\left(\mathrm{C}_{7}\right), 32.3\left(\mathrm{C}_{8}\right), 29.7\left(\mathrm{C}_{3}\right), 26.2\left(\mathrm{C}_{9}\right), 22.8\left(\mathrm{C}_{10}\right), 22.2\left(\mathrm{C}_{14}\right), 16.9\left(\mathrm{C}_{12}\right), 14.2$ $\left(\mathrm{C}_{11}\right), 12.2\left(\mathrm{C}_{15}\right)$.

$m / z, 210\left(\mathrm{MH}^{+}, 89\right), 138(76)$. 
(士)-6-Benzyl-2-methyl-1-propyl-1,2,3,6-tetrahydropyridine (Table 2 entry 8)

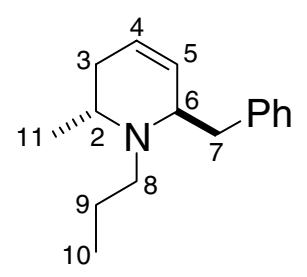

Yellow oil

$85 \%$ yield

$\mathrm{R}_{\mathrm{f}} 0.3$ (hexane:ethyl acetate:triethylamine 94:5:1)

Found: $[\mathrm{M}+\mathrm{H}]^{+} 230.1908 ; \mathrm{C}_{15} \mathrm{H}_{21} \mathrm{~N}+\mathrm{H}$ requires 230.1903 .

$v_{\max } / \mathrm{cm}^{-1}$ (neat) 3028, 2957, 1660, 1604, 1490, 1452, 1371, 1080, 696.

$\delta_{\mathrm{H}}\left(400 \mathrm{MHz} ; \mathrm{CDCl}_{3}\right)$ 7.33-7.18 (m, 5H, $\left.\mathrm{H}_{\mathrm{Ph}}\right), 5.77-5.74\left(\mathrm{~m}, 1 \mathrm{H}, \mathrm{H}_{4}\right), 5.52-5.49(\mathrm{~m}, 1 \mathrm{H}$, $\left.\mathrm{H}_{5}\right), 3.25-3.21\left(\mathrm{~m}, 2 \mathrm{H}, \mathrm{H}_{2}\right.$ and $\left.\mathrm{H}_{6}\right), 2.99$ (dd, $J 6.1$ and 12.9, 1H, $\left.\mathrm{H}_{7}\right), 2.62$ (dd, $J 8.5$ and 12.9, $\left.1 \mathrm{H}, \mathrm{H}_{7}\right), 2.47-2.40\left(\mathrm{~m}, 2 \mathrm{H}, \mathrm{H}_{8}\right), 2.04-1.99\left(\mathrm{~m}, 1 \mathrm{H}, \mathrm{H}_{3}\right)$ 1.91-1.88 (m, $\left.1 \mathrm{H}, \mathrm{H}_{3}\right), 1.41-$ $1.35\left(\mathrm{~m}, 2 \mathrm{H}, \mathrm{H}_{9}\right), 1.09\left(\mathrm{~d}, J\right.$ 6.6, 3H, H $\left.\mathrm{H}_{11}\right), 0.81\left(\mathrm{t}, J 7.3,3 \mathrm{H}, \mathrm{H}_{10}\right) ; \delta_{\mathrm{C}}\left(100.6 \mathrm{MHz} ; \mathrm{CDCl}_{3}\right)$ $140.3\left(\mathrm{C}_{\mathrm{Ph}}\right), 129.6\left(\mathrm{C}_{5}\right), 128.6\left(\mathrm{C}_{\mathrm{Ph}}\right), 128.1\left(\mathrm{C}_{\mathrm{Ph}}\right), 125.9\left(\mathrm{C}_{\mathrm{Ph}}\right), 125.2\left(\mathrm{C}_{4}\right), 59.8\left(\mathrm{C}_{6}\right), 49.0$ $\left(\mathrm{C}_{8}\right), 47.3\left(\mathrm{C}_{2}\right), 40.4\left(\mathrm{C}_{7}\right), 29.8\left(\mathrm{C}_{3}\right), 22.0\left(\mathrm{C}_{9}\right), 17.1\left(\mathrm{C}_{11}\right) 12.0\left(\mathrm{C}_{10}\right)$. $\mathrm{m} / \mathrm{z} 230\left(\mathrm{MH}^{+}, 81\right), 138(100)$. 

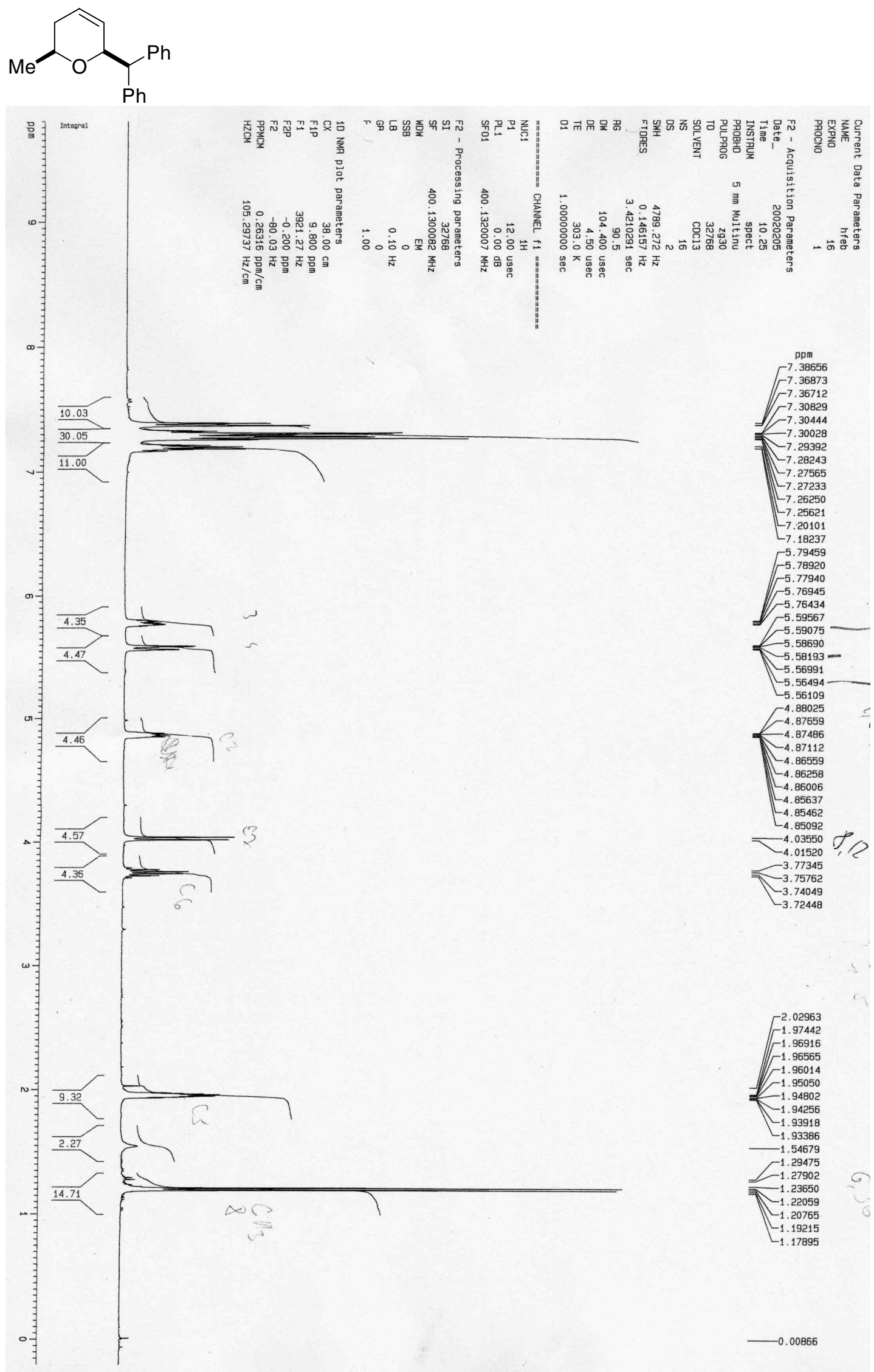

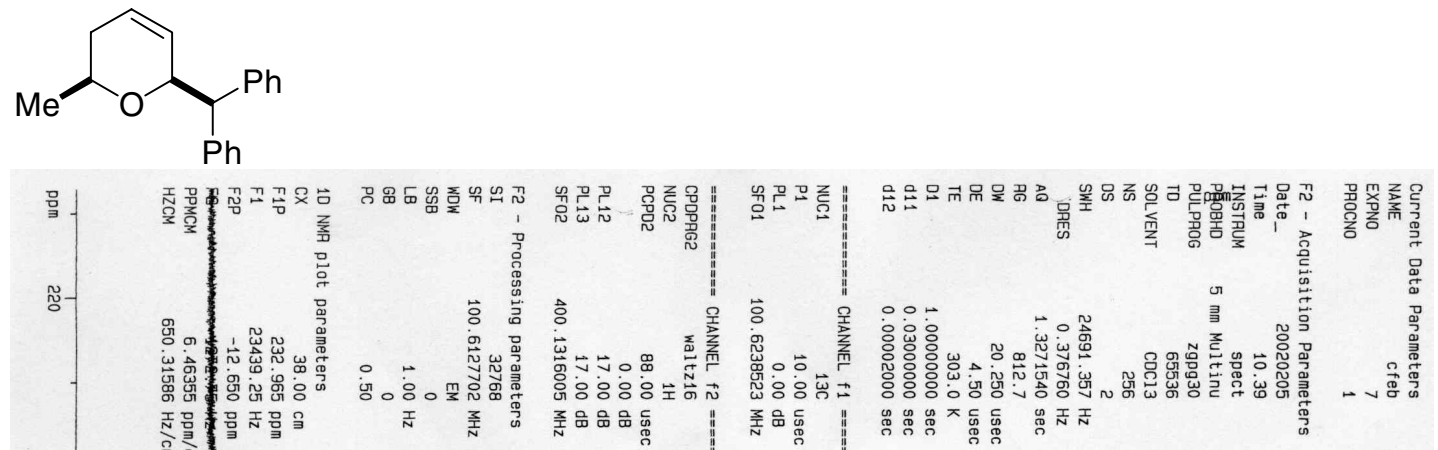

-

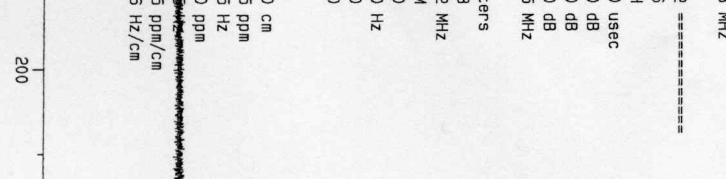

离总

142.502
-142.043

$\overbrace{}^{128.981}$

$-128.362$

127.934
-126.338

-126.053
-125.910

s
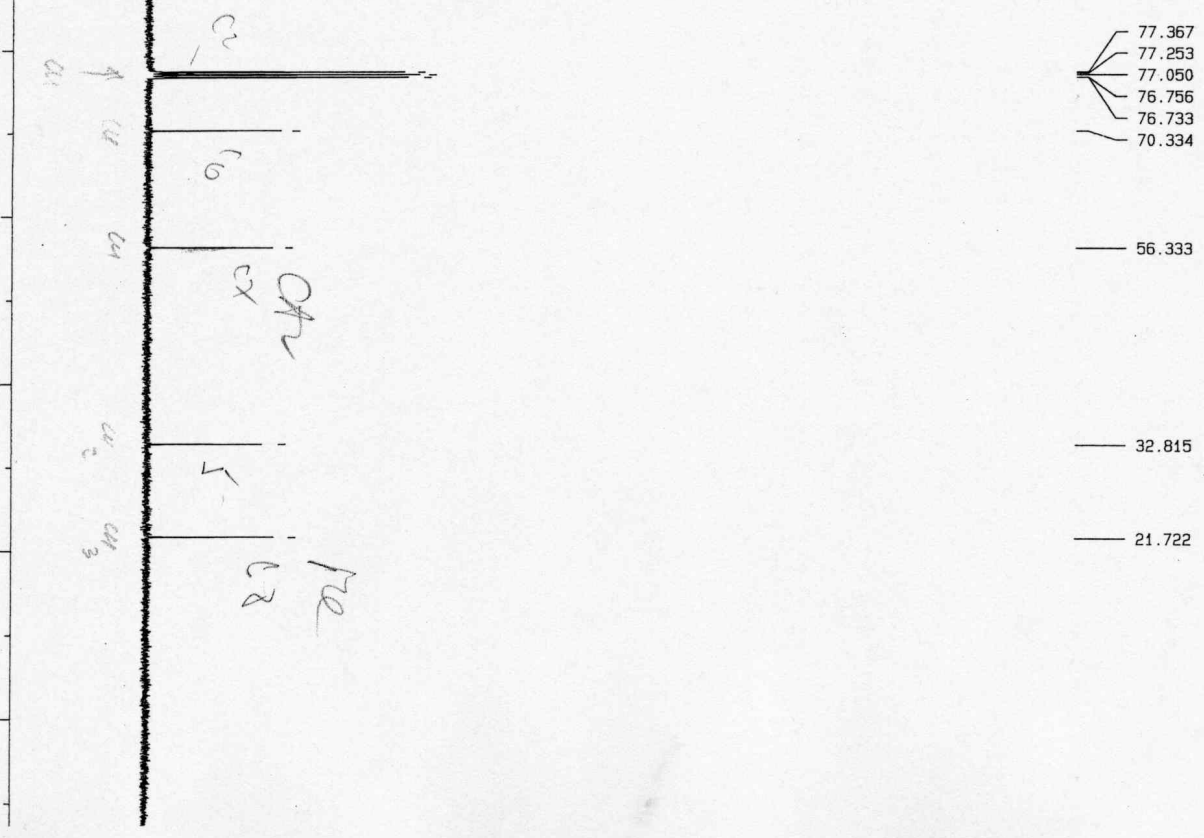
<smiles>CC(=O)O[C@H]1CC(C)OC(C(c2ccccc2)c2ccccc2)[C@H]1OC(C)=O</smiles>

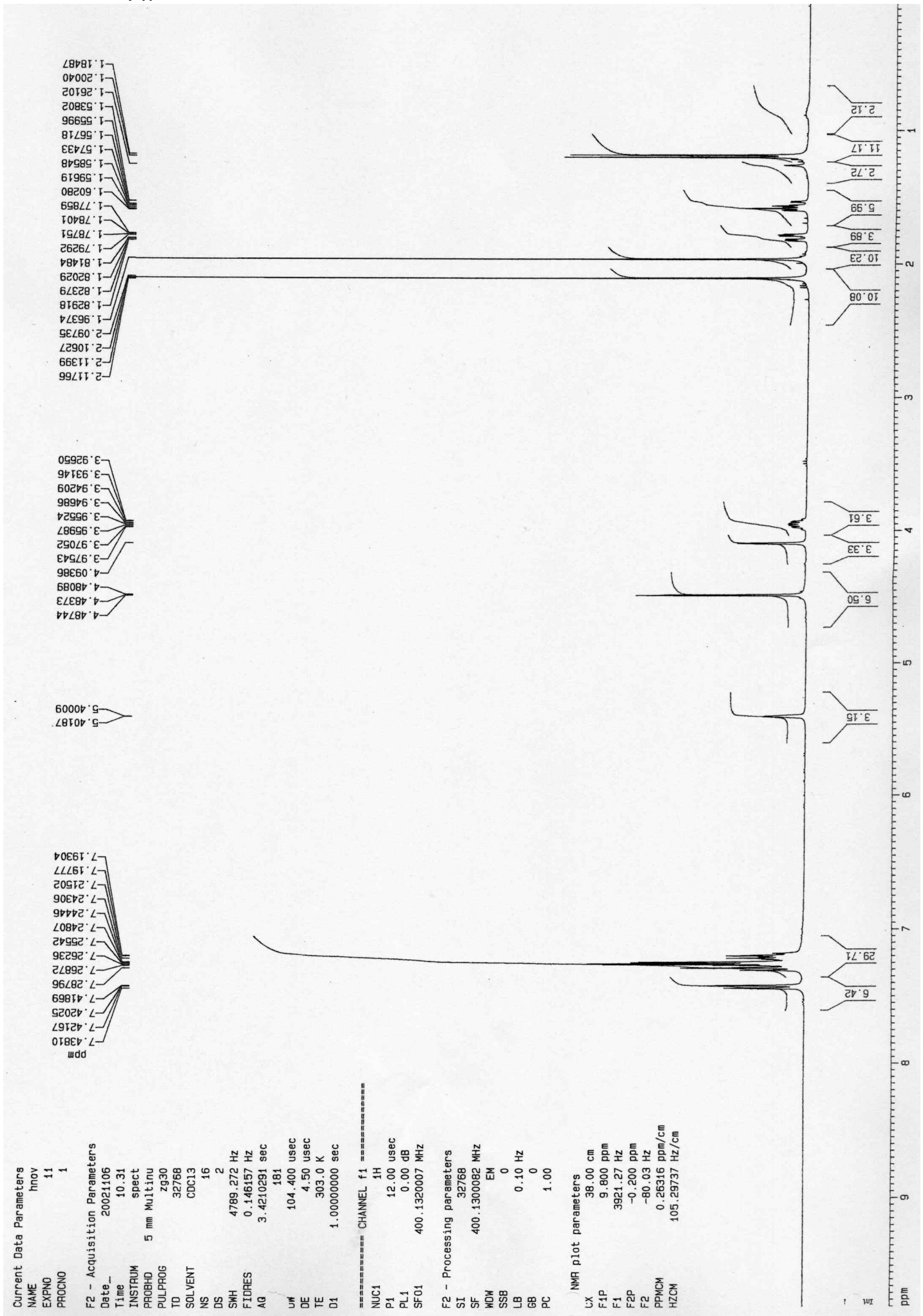


<smiles>CC(=O)O[C@H]1CC(C)OC(C(c2ccccc2)c2ccccc2)[C@H]1OC(C)=O</smiles>

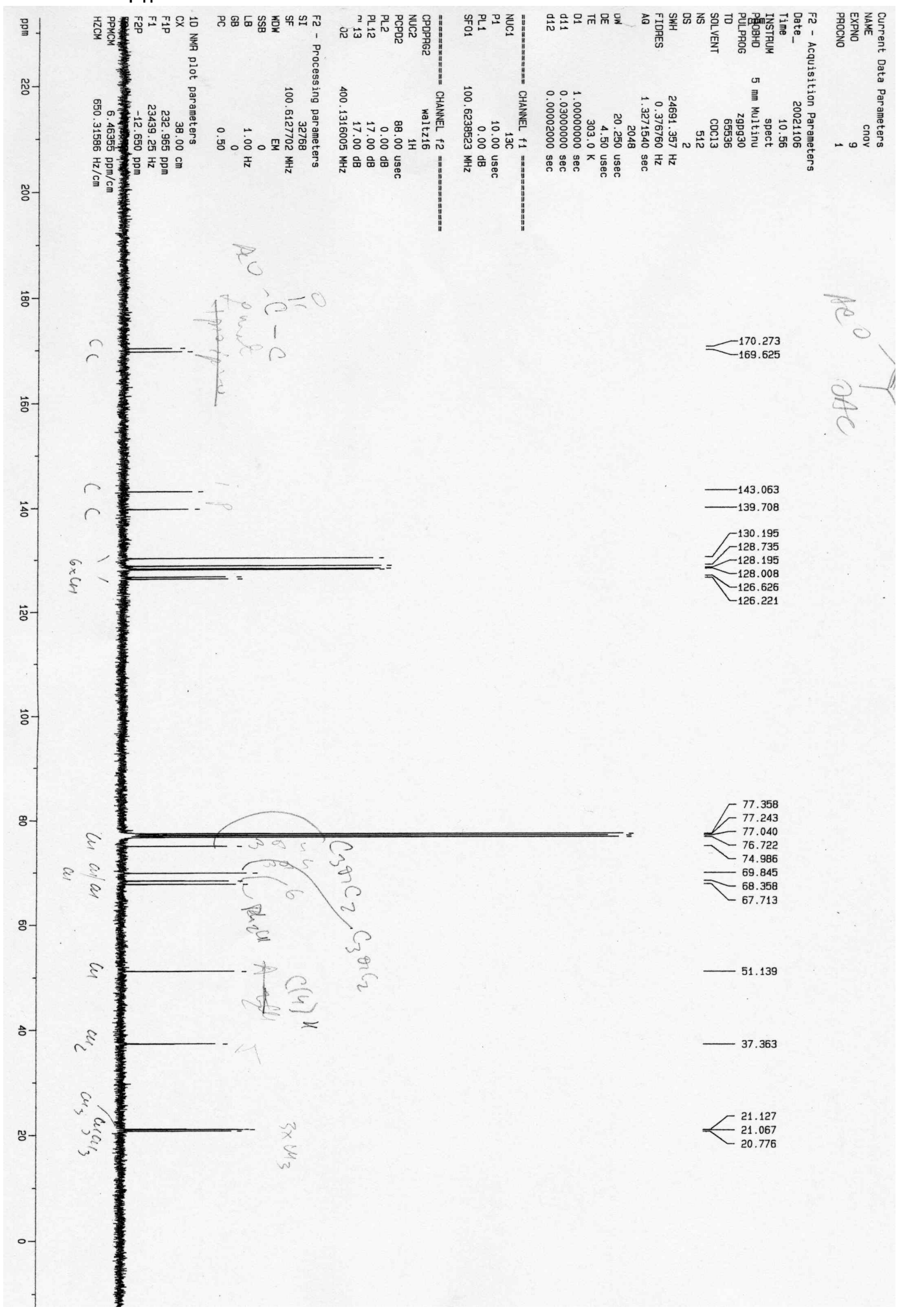



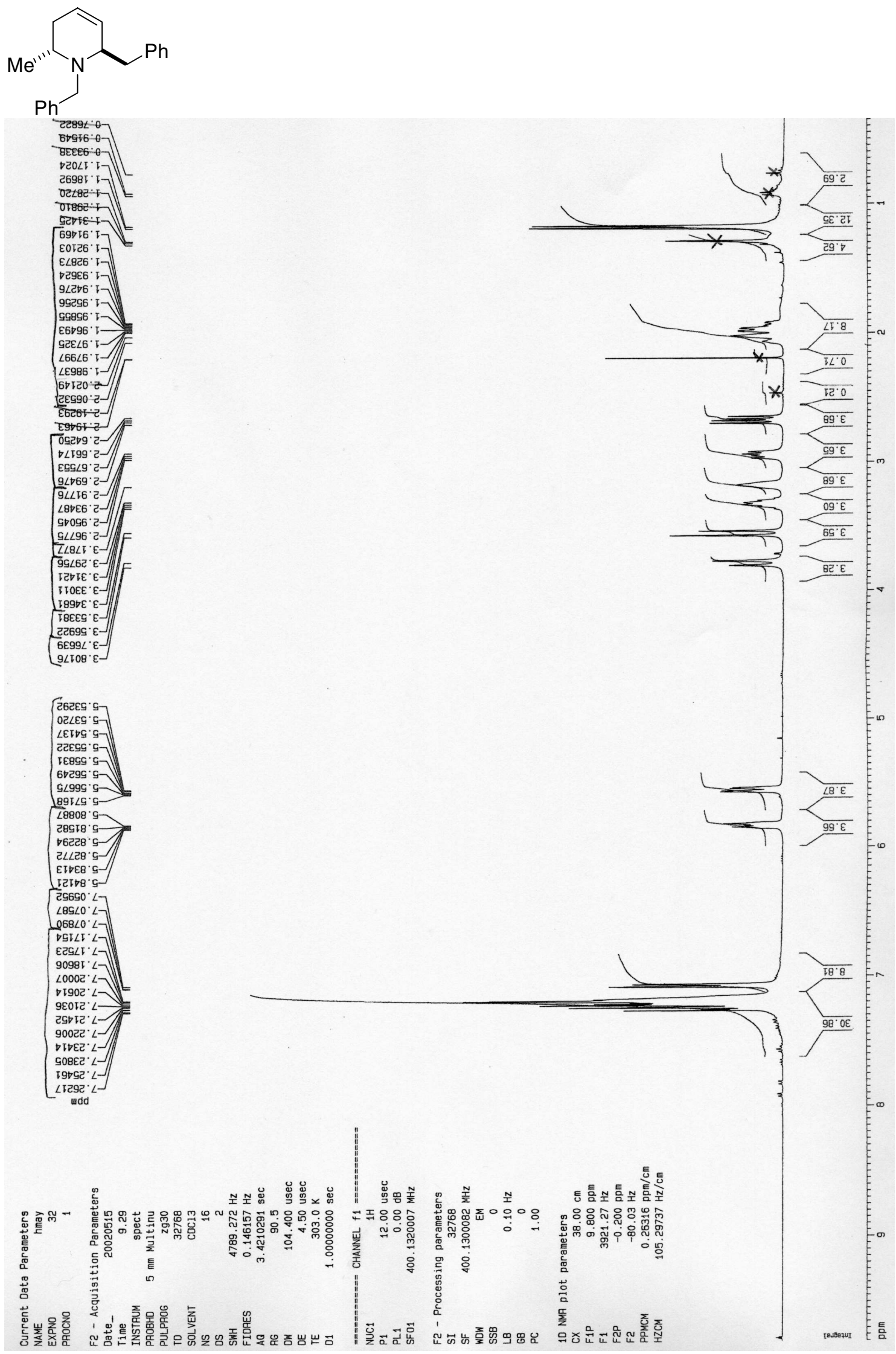
<smiles>C[C@@H]1CC=CC(Cc2ccccc2)N1Cc1ccccc1</smiles>
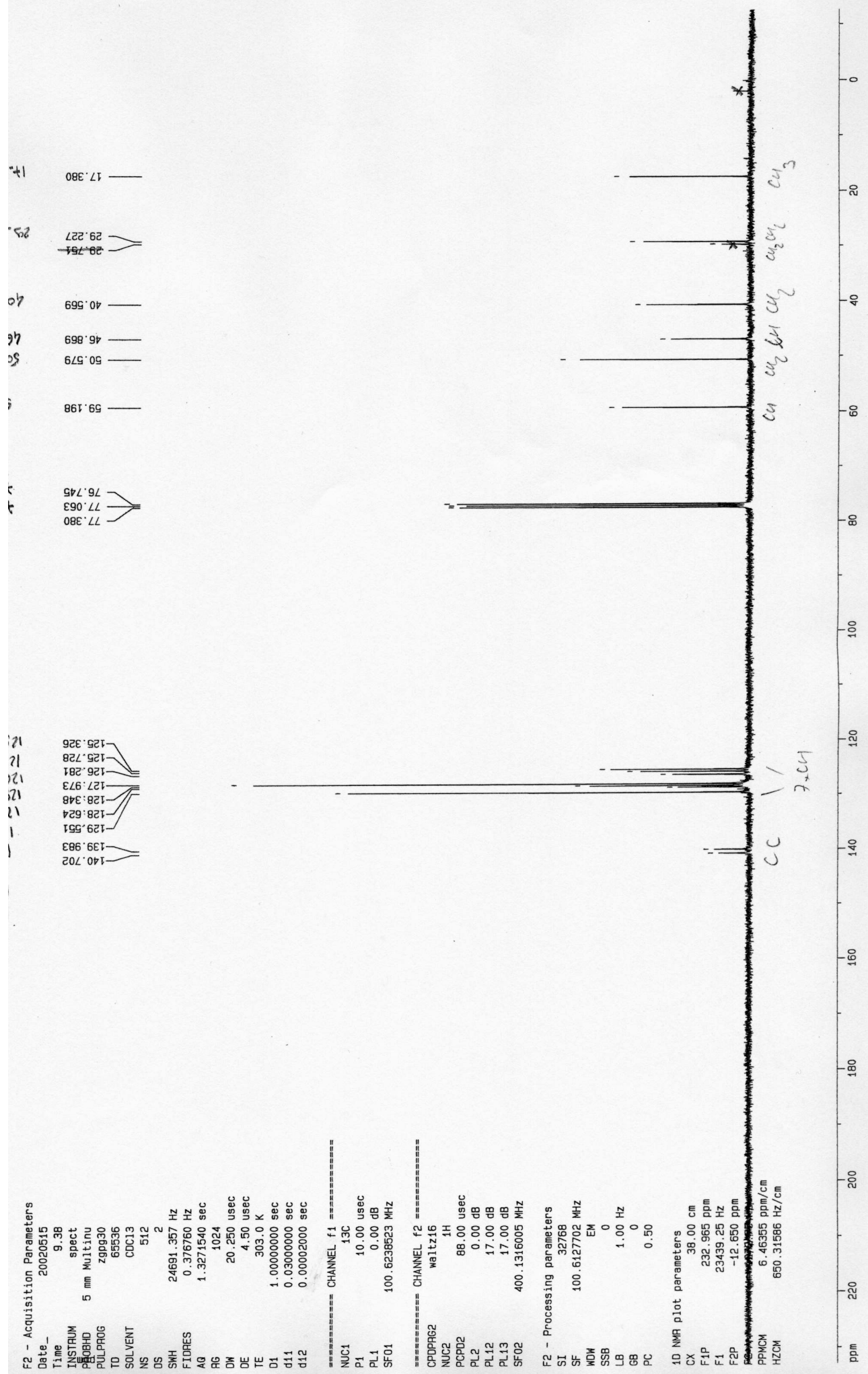